\section{Alcoholic liver disease}

\author{
A D Thomson, G L A Bird, J B Saunders
}

During the 1950 s and ' 60 s when the debate about the relative contribution of alcohol toxicity and malnutrition to alcohol related liver damage was raging in the United States, ${ }^{1}$ Britain was more interested in other forms of liver disease. As awareness of the size of the alcohol problem grew in this country, ${ }^{23}$ research work at the Liver Unit began to reflect the changing situation. Immunology was already well established at King's and the development of a new lymphocytotoxicity assay by Thomson and colleagues provided a useful tool for investigating immunological changes in alcoholic liver disease. ${ }^{4}$ These studies have continued over the past 15 years with the aim of identifying the precise mechanisms involved and why some patients have a propensity to progressive liver damage, even when alcohol is discontinued. ${ }^{5}$

These investigations naturally lead to attempts to identify groups of individuals especially susceptible to alcohol induced liver injury. This was the main focus of research in the 'middle period' from the late 1970s onwards. Research into alcoholic liver disease expanded considerably in 1979 with the formation of a multidisciplinary Alcohol Research Group by Michael Davis and John Saunders. This group extended its work into biochemical mechanisms of liver damage in conjunction with the MRC Human Biochemical Genetics Unit. It also established a programme of research into the underlying alcohol dependency and psychosocial factors. The group, which was supported by the Department of Health and Social Security, forged close links with the Addiction Research Unit at the Maudsley Hospital. This resulted in a more comprehensive approach to management, including psychological as well as medical treatment. ${ }^{6}$

The third main area of work has been in the

Department of Medicine, Greenwich Hospital, London

A D Thomson

\section{Liver Unit, King's}

College School of

Medicine and Dentistry, London

G L A Bird

Centre for Drug and Alcohol Studies, Royal Prince Alfred Hospital and Departments of Medicine and Psychiatry, University of Sydney, Sydney, New South Wales, Australia J B Saunders

Correspondence to: Dr A D Thomson, SE9.
Figure 1: Mechanisms of $T$ and $B$ cell stimulation in alcoholic liver disease. Department of Medicine, Greenwich Hospital, London treatment of alcoholic liver disease. This has included strategies to increase abstinence from alcohol, the effects of nutritional supplementation, pharmacological methods of modifying the disease process and other hepatoprotective agents. Together with Roy Calne in Cambridge the Liver Unit pioneered liver transplantation in Britain and more recently, this method of treatment has been offered to a number of patients with end-stage alcoholic liver damage.

The study of alcoholic liver disease has therefore come of age. The Liver Unit's research programme has developed in response to the increasing problems caused by alcohol in this country. It has evolved logically, using techniques developed for investigating other forms of liver disease, and with an emphasis on collaboration with other units to provide a true multidisciplinary perspective.

\section{Mechanisms of liver damage}

\section{IMMUNOLOGICAL INJURY}

The possibility that alcoholic liver disease might be in part, the result of an 'immune attack' on hepatocytes is suggested by the histological features of alcoholic hepatitis with infiltration of polymorphonuclear and mononuclear cells, and the observation that in some patients progression of the hepatic injury occurred even after abstinence. In severe alcoholic hepatitis especially, liver damage often increases for two to four weeks after admission to hospital and after cessation of alcohol consumption. To determine whether an autoimmune reaction to liver specific proteins was occurring, Cochrane and colleagues ${ }^{7}$ adopted the lymphocytotoxicity assay of Thomson et al. ${ }^{4}$ Lymphocyte toxicity towards normal (rabbit) hepatocytes was shown in 15 of 17 patients with alcoholic hepatitis, and was not present at other stages of alcoholic liver disease. It was possible to block this reaction by the addition of a liver specific protein extracted from the membrane of liver cells, lending support to the view that the exposed protein was acting as an antigen. The probability that lymphocyte mediated toxicity could play an important role in hepatocyte injury in alcoholic hepatitis was further strengthened by the observation that the degree of cytotoxicity correlated well with the presence of liver cell necrosis. Furthermore, antibodies to liver specific protein could be detected in such patients, and correlated with the degree of necrosis. ${ }^{8}$

In an extension of this work, Neuberger and colleagues showed the presence of circulating antibodies in $43 \%$ of patients with alcoholic liver disease which reacted specifically with hepatocytes isolated from ethanol pretreated rabbits. ${ }^{9}$ This suggested that an alcohol related antibody could promote antibody dependent cell medi- 
ated cytotoxicity and react with cell surface antigens resulting from the effects of alcohol or its metabolites on the hepatocyte. Using a similar cytotoxicity system, Crossley et al showed that disulfiram, an inhibitor of acetaldehyde metabolism, enhanced the cytotoxicity, supporting the theory that acetaldehyde, rather than ethanol, was responsible for generating these neoantigens. ${ }^{10}$ Abnormalities of lymphocyte subpopulations in patients with alcoholic liver disease have thrown light on other potential mechanisms of damage. There are defects in helper- $T$ cell function and hyper-reactivity of B cells, accompanied by increased spontaneous production of IgG and IgA."

During this period, therefore, a number of immunological changes were shown which could explain the basis of individual susceptibility to alcoholic liver disease and possibly why liver cell damage continues in some patients after alcohol intake has ceased. A schema is presented to summarise the findings. Patients with alcoholic hepatitis have antibodies reacting with acetaldehyde altered liver cell membrane antigens (Fig 1). Lymphocytes from patients with alcoholic hepatitis are also directly cytotoxic to different target cells in vitro (Fig 2). These mechanisms may act in parallel to cause hepatocyte necrosis. It must be acknowledged, however, that the pathogenetic role of these findings has not been proved, and it is possible that they are the consequence of liver injury which exposes new membrane proteins. A further possibility is that immunological changes act pari passu with other mechanisms.

More recent interest has focussed on the role of cytokines as mediators of hepatocyte damage. In alcoholic liver disease the occurrence of raised concentrations of circulating endotoxin is thought to be because of increased gut permeability or impaired Küpffer cell function. Endotoxin stimulates the release of tumour necrosis factor, a cytokine secreted by monocytes, macrophages, and lymphocytes. Tumour necrosis factor has a wide range of biological actions including the induction of fever, neutrophilia, and hypotension. These are clinical features present in severe alcoholic hepatitis, and in animal models tumour necrosis factor has been implicated as a mediator of liver cell necrosis. Tumour necrosis factor production by peripheral blood mononuclear cells from patients with alcoholic hepatitis has been shown recently to be significantly greater than in controls, both before and after stimulation with endotoxin. ${ }^{12}$ In 21 patients with severe alcoholic hepatitis admitted to the Liver Unit, plasma tumour necrosis factor concentrations were above the normal range in all but one, and significantly higher than in abstinent patients with alcoholic cirrhosis and alcoholics without liver disease. Furthermore, plasma tumour necrosis factor was higher in patients who subsequently died and correlated significantly with bilirubin, fever, and neutrophilia. The concentrations decreased in patients who recovered. ${ }^{13}$ Endotoxins are also implicated in the pathogenesis of hepatic injury. ${ }^{14}$ Although there was no correlation between tumour necrosis factor and endotoxin concentrations in our subjects, endotoxin con-

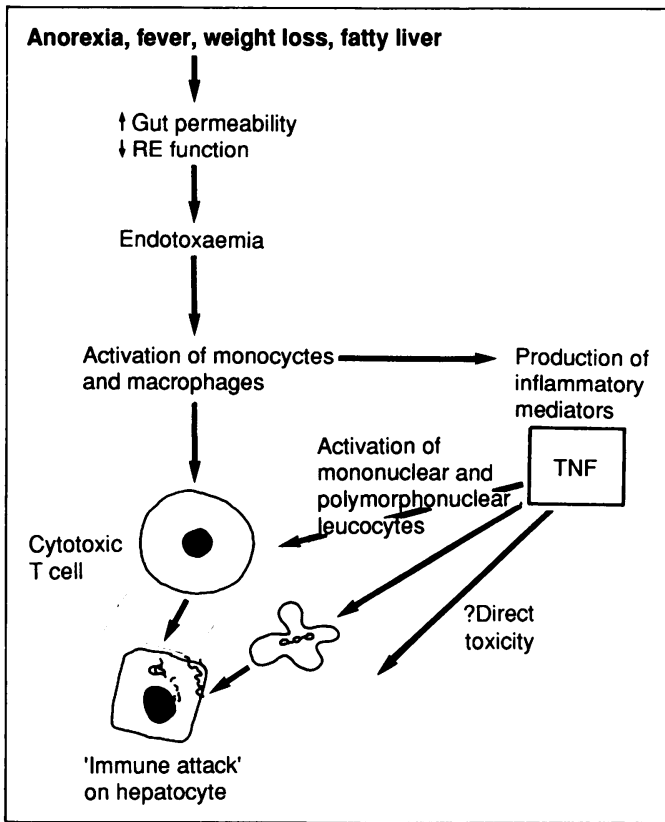

Figure 2: Cell mediated 'immune attack' on hepatocytes could be promoted by endotoxaemia and activation of the cytokine cascade.

centrations were highest in patients with alcoholic hepatitis. Endotoxins could therefore have a synergistic role in hepatocyte damage. ${ }^{14}$

Other cytokines that could be responsible for the clinical manifestations, and/or contribute to the abnormalities seen in cell mediated and humoral immune systems include interleukin-1 and interleukin-2. Raised interleukin-1 activity is associated with fibrosis and cirrhosis. Concentrations are, however, normal in patients with alcoholic hepatitis in whom underlying cirrhosis is not present. ${ }^{15}$ In contrast, interleukin-2 production by peripheral blood mononuclear cells is reduced in inactive alcoholic cirrhosis, and could be one of the factors that contributes to the impaired immune response and susceptibility to infection seen in patients with alcoholic cirrhosis. ${ }^{15}$

BIOCHEMICAL MECHANISMS OF LIVER DAMAGE

The interaction between alcohol, malnutrition, and liver injury has proven to be complex,

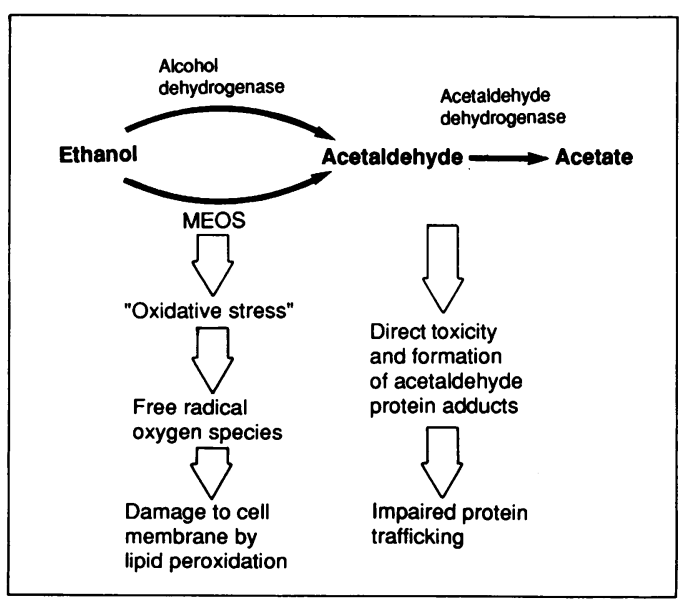

Figure 3: Ethanol metabolism causes increased formation of free radical oxygen species and impaired protein trafficking. 
particularly when considering the evolution of this process in the patient. Although the direct toxic effect of the products of alcohol metabolism is no longer questioned, there is evidence indicating that malnutrition may be needed to produce the spectrum of alcoholic liver damage seen.

In man, ethanol is largely metabolised by two systems: the alcohol dehydrogenase and MEOS pathways, the latter being induced by high levels of alcohol intake. All pathways produce acetaldehyde as an intermediate product. This molecule is extremely reactive, combining with lysine or valine residues or with nucleic acids or peptides, and may produce immunologically foreign proteins or loss of enzyme activity causing the hepatocyte to become less efficient.

Acetaldehyde also reacts with tubulin, interfering with its polymerisation by binding covalently to its dimer form. This impedes the movement of organelles and proteins within the cell and the interference with hepatocyte cell membrane proteins is likely to impair protein transport across the cell surface membrane. These findings may explain why high ethanol intake causes accumulation of protein within the hepatocyte. Damage to microtubules or their associated proteins will interrupt protein transport, and membrane protein damage is consistent with the impairment of receptor mediated endocytosis of macromolecules in the liver in alcoholism. Defects in protein trafficking are likely to interfere with the function of many hormones, trophic factors, biogenic peptides, and carrier proteins. ${ }^{16}$ The oxidation of acetaldehyde especially by the low $\mathrm{Km}$ acetaldehyde dehydrogenase will generate free radical oxygen species (Fig 3). These molecules are highly reactive and are capable of damaging cells.

Studies into biochemical mechanisms of alcoholic liver injury started in 1980 with the establishment of techniques for assaying acetaldehyde, and the activities and isoenzyme profiles of the principal alcohol metabolising enzymes. Higher blood acetaldehyde concentrations were found after administration of alcohol in alcohol dependent subjects and those with liver damage than in healthy non-alcoholic controls. ${ }^{17}$ Furthermore, hepatic aldehyde dehydrogenase activity was lower in patients with alcoholic cirrhosis than in those with fatty liver. This seems to be an acquired defect and suggests that patients with cirrhosis are particularly liable to accumulate acetaldehyde during metabolism of alcohol. They may experience a vicious circle of increasing acetaldehyde concentrations, causing greater liver injury, which further suppresses aldehyde dehydrogenase activity. ${ }^{18}$

As described previously, alcohol, and specifically its metabolite acetaldehyde, have been shown to result in the formation of neoantigens. These are presumably located on the surface of hepatocytes. To complement this work, the activities of surface membrane associated enzymes were studied. Alcohol was shown to suppress activities of $\left(\mathrm{Na}^{+}-\mathrm{K}^{+}\right)$ATPase in vitro. ${ }^{19}$ Although the concentrations that produce this effect are generally higher than occur in man, these experiments serve to illustrate the multifaceted effects of alcohol and raise the possibility that in vivo acetaldehyde synthesised in proximity to surface membranes may inhibit enzyme and ion channel function at concentrations approximating to those occurring during the metabolism of alcohol.

\section{Susceptibility to alcoholic liver disease}

\section{EPIDEMIOLOGY}

Early epidemiological studies of alcoholic liver disease showed a dose response relationship between cumulative alcohol consumption and the risk of cirrhosis. ${ }^{20}$ In contrast, recent prospective studies have suggested that only about $20 \%$ of heavy drinkers will ultimately develop alcoholic cirrhosis, provided their average daily intake regularly exceeds a threshold dose of about 50-60 g. ${ }^{5}$ This is consistent with the common clinical experience that some individuals develop alcoholic cirrhosis while still in their early 20s while others escape damage after a lifetime of heavy drinking. Investigation of the basis for this differing susceptibility has been a major focus of research in the Liver Unit since the mid-1970s.

Individual variation in susceptibility could be partly explained by environmental factors, such as alteration of the metabolic handling of alcohol by exposure to enzyme inducing drugs, or concomitant infection with hepatitis viruses. It might reflect subtle differences in the pattern of drinking between individuals. It is also likely that genetic factors play a significant role. ${ }^{18}$

Initial studies examined the influence of gender and histocompatibility antigen (HLA) status. In the first large clinical series reported from the Liver Unit, Krasner and colleagues ${ }^{21}$ showed that women were more likely to have alcoholic hepatitis at presentation than men; $91 \%$ of men had an average daily alcohol intake exceeding $150 \mathrm{~g}$ compared with $65 \%$ of women.

This substantial difference in susceptibility between the sexes was confirmed in a later series by Saunders and colleagues..$^{22}$ The earlier report $^{22}$ synthesised the published work on sex related differences, and showed that women developed alcoholic liver disease at a younger age, after a shorter period of drinking, and at a lower mean daily alcohol intake than men. They formulated three hypotheses: (i) differences in body composition, specifically the lower lean body mass and higher proportion of fatty tissue in women resulted in higher blood alcohol concentrations after a standard drink than in men, and accordingly more severe tissue injury; (ii) the greater propensity of women to develop autoimmune disorders predisposed them to develop a heightened immune response to normal liver cell components and to alcohol induced neoantigens (this might also be related to histocompatibility antigen factor status); (iii) that sex related differences in alcohol metabolising enzymes resulted in women having higher acetaldehyde concentrations and a greater risk of free radical injury.

The last hypothesis was more speculative, although alcohol dehydrogenase activity is higher in the females of certain animal species than in males. 
The study by Bailey and colleagues in $1976^{24}$ was the first to draw attention to an histocompatibility antigen factor association with alcoholic cirrhosis: $45 \%$ of such patients had the histocompatibility antigen factor B8, compared with $25 \%$ of healthy controls and $10 \%$ of patients with alcoholic fatty liver. Because of the large number of histocompatibility antigen factor antigens tested, it was possible that this apparently significant difference could have arisen by chance. A later study from the Royal Free Hospital, however, in which only the presence of B8 was tested confirmed the association specifically in males. ${ }^{25}$ Similar findings were reported from some overseas centres but not in others. ${ }^{26}$ Interpretation of these studies is difficult because a high prevalence of a particular histocompatibility antigen factor marker could simply reflect a predisposition to alcohol abuse.

In 1979 the Alcohol Research Group developed a standardised protocol for the assessment of all patients with alcoholic liver disease admitted to the Liver Unit. ${ }^{18}$ This included a comprehensive assessment of lifetime alcohol intake, alcohol dependence and related problems, histocompatibility antigen factor status, hepatitis serology, markers of immune reactivity, liver histology, and in subsamples the isoenzyme profile of alcohol dehydrogenase and aldehyde dehydrogenase. In addition, follow up data were collected for the first two years after discharge. A computerised data base was established to facilitate analysis. Over 400 patients were systematically investigated over the next five years.

In this way Saunders and colleagues were able to analyse the effect of histocompatibility antigen factor status in relation to cumulative alcohol consumption in a large group of patients with alcoholic cirrhosis. Both male and female patients with the histocompatibility antigen factor B8 antigen had been drinking in excess of $40 \mathrm{~g}$ alcohol per day for a shorter period of time than patients with cirrhosis of comparable degree without histocompatibility antigen factor B8. ${ }^{23}$ The mean daily alcohol intake of the two groups was the same, showing that genetic determinants linked to the antigen were not influencing drinking behaviour. Histological signs of alcoholic hepatitis and alcoholic hyaline were more common in the histocompatibility antigen factor B8 group. It was suggested that alcohol induced antigens might result in a heightened immune response in patients with B8 and thereby favour the development of alcoholic hepatitis and progression to cirrhosis. A particularly striking finding in this study was that female patients had developed cirrhosis in just over half the time of male patients. Studies currently underway in the Liver Unit and other centres, using molecular biology techniques, will provide further clues on genetic differences in immunological and enzyme phenotypes.

Collaborative work with Dr David Hopkinson at the MRC Human Biochemical Genetics Unit in London lead to the development of a novel technique for the simultaneous electrophoretic separation of isoenzymes of alcohol dehydrogenase and aldehyde dehydrogenase in human liver biopsy specimens. ${ }^{27}$ Isoenzyme profiles were determined in 85 patients with different grades of alcoholic liver injury. No differences were found in the prevalence of the phenotypes of alcohol dehydrogenase. A reduction in activity of the mitochondrial isoenzyme of aldehyde dehydrogenase was, however, found in patients with alcoholic cirrhosis. This was considered to be an acquired rather than an inherited defect, but one that could lead to progressive liver injury mediated by acetaldehyde. ${ }^{18}$

The role of environmental factors other than alcohol has been the subject of much debate. Although the majority of patients with alcoholic cirrhosis have evidence of malnutrition, it appears that this is caused predominantly by the effects of the disease process. It is reasonable to suppose that alcohol might act in synergy with other hepatotoxic agents to cause disease which would not occur if either were acting independently. Alcohol interacts with paracetamol to exacerbate hepatic necrosis, but no drugs were found to be consistently associated with a particular type of damage in the Liver Unit series. Evidence of previous infection with hepatitis B virus was found in $20 \%$ of alcoholic cirrhotic patients, but this was not significantly different from those with fatty liver. ${ }^{28}$

Important clues to the nature of the underlying drinking behaviour were obtained from the comprehensive alcohol assessment. Nearly all patients with alcoholic hepatitis or cirrhosis had a history of unremitting daily drinking, in contrast to those with fatty liver or fibrosis, whose drinking was often of the binge type. Dependence was assessed by using the Severity of Alcohol Dependence Questionnaire, developed at the Addiction Research Unit. ${ }^{29}$ Wodak and colleagues found scores for dependence were relatively low among patients with alcoholic hepatitis or cirrhosis..$^{30}$ Most patients had never experienced severe physical dependence symptoms, which suggests that their drinking does not reach the heights of the typical 'alcoholic'. It is well integrated into the person's life so that a high intake can be sustained for many years. Only a minority of patients had received advice about reducing or ceasing drinking in the past, and only $22 \%$ had undergone formal treatment. ${ }^{3}$ Those who had done so were the more severely dependent or those with major psychosocial problems..$^{31}$ Again, it suggests that the drinking pattern of the typical patient with severe alcohol induced liver disease does not identify that individual as an alcoholic requiring treatment.

\section{Treatment of alcoholic liver disease}

\section{TREATMENT OF THE DRINKING PROBLEM}

Abstinence remains the most important single measure to improve the prognosis of patients with alcoholic liver disease. In studies from the Liver Unit and elsewhere, the five year survival of patients who stop drinking is substantially greater than those who continue. ${ }^{21}$ This is true, irrespective of whether the patient is still compensated or has signs of decompensation. The analysis by Krasner $e t$ al was among the first to highlight the significantly greater mortality and younger age of death in women who continue to 
drink, which was shown to correlate with their increased tendency to histological progression of the disease if alcohol is not withdrawn. ${ }^{21}$ It was also shown that women are more likely to develop progression of alcoholic liver disease even if they stop drinking.

Rates of abstinence after discharge range from $4 \%$ to $30 \%$ in most series. To try to improve on this, investigations into psychiatric disorders and psychosocial impairment were instituted in 1980. Forty eight per cent of patients with alcoholic liver disease had a history of depressive disorder compared with $22 \%$ of patients with non-alcoholic liver disease of comparable severity..$^{32}$ In women there was a two fold higher rate of neurotic disorders than in the nonalcoholic control group. These findings had a direct influence on the treatment of the underlying drinking behaviour. As well as receiving advice on abstinence and feedback of the results of the medical assessment, patients were screened for psychiatric problems. Referral was arranged for those who had such disorders and some of the therapies used in the Alcohol Problems Service at the Maudsley Hospital were adopted by the staff of the Alcohol Research Group. Although a controlled trial of this combined approach was not undertaken, abstinence rates of up to $55 \%$ were achieved, with validation by monitoring of urine samples for the presence of alcohol. ${ }^{33}$

\section{PHARMACOLOGICAL TREATMENT OF ALCOHOLIC LIVER DISEASE}

The mortality from alcoholic liver disease in the first three months after admission to hospital varies between $22 \%$ for all histological categories of patient to $60 \%$ for those with severe alcoholic hepatitis. Specific forms of treatment for alcoholic liver disease have been evaluated in patients with alcoholic hepatitis in an attempt to decrease short term mortality and prevent progression to cirrhosis. In this area the Liver Unit has been particularly active.

The possibility that immune mechanisms play a role in the pathogenesis of alcoholic hepatitis encouraged controlled trials of corticosteroids on the basis of their immunosuppressant and antiinflammatory activity. The first study of corticosteroids in acute severe alcoholic hepatitis was reported by Helman and colleagues, who showed a statistically significant improvement in short term mortality with $40 \mathrm{mg} /$ day prednisolone in 14 severely ill patients, all of whom had hepatic encephalopathy. ${ }^{34}$ In a controlled trial in the Liver Unit, 55 patients were randomised to receive either methylprednisolone $1 \mathrm{~g}$ daily for three days or placebo, on the basis that this dose of steroids was known to be effective in reversing acute rejection in renal and liver transplants. There was no significant difference, however, in mortality between the treatment and placebo groups, with $57 \%$ of the control group and $63 \%$ of the treatment group dying during the study. ${ }^{35}$

Interest in the use of steroids in the treatment of alcoholic hepatitis has continued and there have been 10 other randomised controlled trials in the last 20 years. A recent meta-analysis of the 11 trials containing sufficient information suggested that in the small subgroup of very ill patients with spontaneous hepatic encephalopathy, in whom sepsis is not present, steroids could be of significant benefit. ${ }^{36}$

Among other specific therapies for alcoholic hepatitis, the use of nutritional supplements has been proposed on the basis that nutritional deficiencies may promote the toxic effects of alcohol by the depletion of amino acids and enzymes. ${ }^{37}$ Evidence for this comes from a large Veterans Administration study in which all patients with alcoholic hepatitis had evidence of protein-calorie malnutrition and in which the severity of liver injury correlated with the degree of malnutrition. ${ }^{38}$ The current prevalence of malnutrition in British patients with alcoholic hepatitis is probably not as high (C Wicks, personal communication) although in a survey of nutritional status of patients admitted to the Liver Unit, with all categories of alcoholic liver disease, published in 1980, malnutrition was present in over $55 \%$, more than in patients with non-alcoholic cirrhosis. ${ }^{39}$

A number of studies have evaluated supplementation with intravenous aminoacids in alcoholic hepatitis. The largest of these was performed by Calvey and colleagues in the Liver Unit, in which 64 patients were treated with either conventional protein supplements rich in branched chain aminoacids or an unsupplemented diet. ${ }^{40}$ Positive nitrogen balances were achieved with supplements containing $10 \mathrm{~g}$ or more nitrogen/day, but no differences in mortality were seen between the three treatment groups. Mortality overall was $32-43 \%$. In none of the patients did exogenous nitrogen precipitate hepatic encephalopathy. More recent trials of parenteral aminoacid supplements have not shown any improvement in mortality with treatment; other therapeutic approaches are therefore under scrutiny. ${ }^{41}$

Hepatoprotective agents have also been investigated, the rationale being that hepatocyte damage, secondary to intracellular metabolic changes arising from alcoholic metabolism, such as the generation of free radical oxygen species, could be prevented. A recent placebo controlled trial has examined polyunsaturated phosphatidyl choline. This has been shown in animal models to improve membrane fluidity and enhance active transport activity. In a double blind randomised trial, 53 patients with alcoholic hepatitis were treated with polyunsaturated phosphatidyl choline and 51 with placebo for two years. The outcome was assessed at the end of the treatment period in terms of mortality, histological changes and biochemistry. Unfortunately, no statistically significant differences were seen. ${ }^{42}$

\section{LIVER TRANSPLANTATION IN ALCOHOLIC LIVER DISEASE}

Several reports now show that survival after transplantation for alcoholic liver disease is as good as it is for other forms of chronic liver disease. In the King's College Hospital and Cambridge series, the outcome in 24 patients transplanted for alcoholic cirrhosis between 1980 and 1990 has recently been reviewed. ${ }^{43}$ In these 
patients one year survival was $66 \%$, actually better than in patients transplanted for other forms of cirrhosis. In most, the indication for transplantation was repeated admission to hospital for complications of portal hypertension (ascites, variceal bleeding and hepatic encephalopathy), but in six patients the indication was hepatocellular carcinoma superimposed on cirrhosis.

Rehabilitation, as defined by a return to occupational and social functioning was evident in 17 of the 18 patients who survived at least three months. Three patients were suspected of relapsing to heavy drinking within six months after transplant. Although they did not admit to taking alcohol, there was corroborative evidence of a return to drinking shown by rises in mean cell volume and a raised gammaglutamyl transferase to alkaline phosphatase ratio. In one of these patients, liver histology two years after transplant showed alcoholic hyaline, fatty change, and advanced fibrosis, highly suggestive of alcohol induced damage. It is of considerable interest that this patient is positive for histocompatibility antigen factor B8, which was linked to earlier work showing a more rapid development of cirrhosis. ${ }^{23}$ As the graft was of a completely different histocompatibility antigen factor type, this could be evidence that the vulnerability of the liver to alcohol in an histocompatibility antigen factor $\mathrm{B} 8$ positive individual is secondary to host factors rather than to characteristics intrinsic to the liver. The three patients who returned to harmful drinking were the only ones not abstinent before transplant.

Analysis of the indications for transplantation reveals some differences in the selection criteria used by British and American groups. In the Cambridge and King's College Hospital series, all except three patients were abstinent for at least six months at the time of transplantation and therefore fulfilled the criteria of the National Institute of Health Consensus Conference. ${ }^{44}$ In the recent report on liver transplantation in alcoholic liver disease from Pittsburgh, patients were selected on the basis of their 'urgency of need' as assessed by the severity of the patient's medical condition, and a minimum period of abstinence was not required. ${ }^{45}$ Of the 73 patients in the Pittsburgh series, none have returned to harmful drinking after transplantation, although follow up was conducted by telephone survey only, and a comparison of mean cell volume and serum enzyme activities, which could have pointed to harmful drinking, was not made. ${ }^{46}$ In contrast to the American study, our results suggest that liver transplantation should not be considered as a treatment for alcohol dependence in itself, and the criteria for selecting patients under normal circumstances should include a period of abstinence of at least six months.

\section{Conclusion}

Research in the Liver Unit during the past 25 years has been at the forefront of intellectual endeavour. Major contributions in the field of alcoholic liver disease include studies of immunological mechanisms to determine why some patients are more susceptible to aggressive liver damage at a cellular level. There has been considerable effort devoted to identify groups of susceptible individuals and, in particular, there has been the recognition that women, those with the histocompatibility antigen factor B8 antigen and with an unremitting pattern of daily drinking are at particular risk. The Liver Unit has undertaken many studies to find substances which would ameliorate the outcome of alcoholic hepatitis and it is unfortunate that success has been elusive. The extensive expertise in liver transplantation, however, now available to patients with alcoholic liver disease, will no doubt contribute substantially in the future. Attempts to identify the significant steps in alcohol induced hepatocyte necrosis and the factors influencing aggressive histological deterioration in alcoholic liver disease have generated much work over the past quarter of a century. It is likely that alcoholic liver disease arises from an interplay between biochemical and immunological disturbances. There are several genetic and environmental factors, including malnutrition, which are important in determining the propensity to develop cirrhosis and these factors, together with their degree of influence, may vary at different stages of the disease process. Much work, therefore, remains to be done, but with new information, new techniques and a record of continued commitment, the Liver Unit is well placed to make important contributions in this area in the future.

1 Thomson AD. A silver jubilee. Alcohol Alcohol 1990; 25: 91-3. 2 Saunders JB, Walters JRF, Davies P, Paton A. A 20-year prospective study of cirrhosis. Br Med F 1981; 282: 263-6. 3 Evans $M$. The Medical Council on Alcohol and its international journal. Alcohol Alcohol 1990; 25: 95-102.

4 Thomson AD, Cochrane AMG, McFarlane IG, Eddleston ALWF, Williams R. Lymphocyte cytotoxicity to isolated hepatocytes in chronic active hepatitis. Nature 1974; 252: 721 .

5 Bird GLA, Williams R. Factors determining cirrhosis in alcoholic liver disease. Mol Aspects Med 1988; 10: 97-105. 6 Saunders JB. What future for the multidisciplinary approach to alcohol problems? BrF Addict 1984; 79: 129-30.

7 Cochrane AMG, Moussouros A, Portmann BP, et al. Lymphocyte cytotoxicity for isolated hepatocytes in alcohol liver disease. Gastroenterology 1977; 72: 918-23.

8 Perperas A, Tsantoulas D, Portmann B, Eddleston ALWF, Williams R. Autoimmunity to a liver membrane lipoprotein and liver damage in alcoholic liver disease. Gut 1981; 22: 149-53.

9 Neuberger JM, Crossley IR, Saunders JB, et al. Antibodies to alcohol altered liver cell determinants in patients with alcoholic liver disease. Gut 1984; 25: 300-4.

10 Crossley IR, Neuberger JM, Davis M, Eddleston ALWF, Williams R. Ethanol metabolism in the generation of new antigenic determinants on liver cells. Gut 1986; 27: 186-9.

11 Nouri-Aria KT, Alexander GJM, Portmann BP, Hegarty JE, Eddleston ALWF, Williams $R$. T and $B$ cell function in alcoholic liver disease. $\mathcal{F}$ Hepatol 1986; 2: 195-207.

$12 \mathrm{McClain}$ CJ, Cohen DA. Increased tumour necrosis factor production by monocytes in alcoholic hepatitis. Hepatology 1989; 9: 349-51.

13 Bird GLA, Sheron N, Goka AJ, Alexander GJM, Williams R. Increased plasma tumour necrosis factor in acute alcoholic hepatitis. Ann Intern Med 1990; 112: 917-20.

14 Nolan JP. Intestinal endotoxins as mediators of hepatic injury - an idea whose time has come again. Hepatology 1989; 10: - an idea

15 Bird GLA, Nouri-Aria K, Daniels H. Alexander GJM, Williams $R$. Contrasts in interleukin-1 and interleukin-2 activity in alcoholic hepatitis and cirrhosis. Alcohol Alcohol 1989; 24: 541-6.

16 Pratt OE, Roopari HK, Shaw GK, Thomson AD. The genesis of alcoholic brain tissue injury. Alcohol Alcohol 1990; 25: 217-30.

17 Clow A, Tophan A, Saunders JB, Murray RM, Sandler M. The role of salsolinol in alcohol intake and withdrawal. Prog Clin Biol Res 1985; 183: 101-3.

18 Saunders JB, Williams R. The genetics of alcoholism. Alcohol Alcohol 1983; 18: 189-217.

19 Gonzalez-Calvin JL, Saunders JB, Williams R. Effects of alcohol and acetaldehyde on hepatic plasma membrane ASPases. Biochem Pharmacol 1983; 32: 1723-8. 
20 Lelbach WK. Cirrhosis in the alcoholic and its relation to the volume of alcohol abuse. Ann NY Acad Sci 1975; 252: 85-105.

21 Krasner N, Davis M, Portmann B, Williams R. Changing pattern of alcoholic liver disease in Great Britain: relation to pattern of alcoholic liver disease in Great Britain: relation to

22 Saunders JB, Davis M, Williams R. Do women develop alcoholic liver disease more readily than men? $\mathrm{Br}$ Med $\mathscr{f}$ 1981; 282: 1140-3.

23 Saunders JB, Wodak AD, Haines A, et al. Accelerated development of alcoholic cirrhosis in patients with HLA B8. Lancet 1982; i: $1381-4$.

24 Bailey RJ, Krasner N, Eddleston ALWF, et al. Histocompatability antigens, autoantibodies and immunoglobulins in alcoholic liver disease. $\mathrm{Br} M e d$ F 1976; ii: 727-9.

25 Morgan MY, Ross MGR, Ng CM, Adams DM, Thomas HC Sherlock S. HLA-B8, immunoglobulins, and antibody response in alcohol-related liver disease. $\mathcal{F}$ Clin Pathol 1980; 33: 488-92.

26 Eddleston ALWF and Davis M. Histocompatability antigens in alcoholic liver disease. Br Med Bull 1982; 38: $13-6$.

27 Ricciardi BR, Saunders JB, Williams R, Hopkinson DA. Identification of alcohol dehydrogenase and aldehyde dehydrogenase isoenzymes in human liver biopsy specimens. Clin Chim Acta 1983; 130: 85-94.

28 Saunders JB, Wodak AD, Morgan-Capner $\mathrm{P}$, et al. Importance of markers of hepatitis $B$ virus in alcoholic liver disease. BrMed F 1983; 286: 1851-4.

29 Stockwell T, Hodgson R, Edwards G, Taylor C, Rankin H The development of a questionnaire to measure severity of alcohol dependence. Br F Addict 1979; 74: 79-87.

30 Wodak, AD, Saunders JB. Ewusi-Mensah I, Davis M, Williams $R$. Severity of alcohol dependence in patients with alcoholic liver disease. $\mathrm{BrMed}$ F 1983; 287: 1420-2.

31 Saunders JB, Wodak AD, Williams R. Past experience of advice and treatment for drinking problems of patients with alcoholic liver disease. $B r \mathcal{F}$ Addict $1985 ; 80: 51-6$.

32 Ewusi-Mensah I, Saunders JB, Williams R. The clinical nature and detection of psychiatric disorders in patients with alcoholic liver disease. Alcohol Alcohol 1984; 19: 297-302.
33 Westaby D, Macdougall BRD, Saunders JB, Williams R. A study of risk factors in patients with cirrhosis and varicea bleeding. In: Westaby D, Macdougall BRD, Williams R, eds. Variceal bleeding. London: Pitman Medical, 1982: 21-33.

34 Helman RA, Temko MH, Nye SW, Fallon HJ. Alcoholic hepatitis: natural history and evaluation of prednisolone and $1971 ; 74: 341-21$.

35 Theodossi A, Eddleston ALWF, Williams R. Controlled tria of methylprednisolone therapy in severe acute alcoholic hepatitis. Gut 1982; 23: 75-9.

36 Reynolds TB, Benhamou JP, Blake J, Naccarto R, Orrego H. Treatment of alcoholic hepatitis. Gastroenterology International 1989; 2: 208-16.

37 Sherlock S. Nutrition and the alcoholic. Lancet 1984; i: 436-8.

38 Mendenhall CL, Anderson S, Weesner RE, Goldberg SJ, Crolic KA. Protein-calorie malnutrition associated with alcoholic hepatitis. Am $₹$ Med 1984; 76: 211-22.

39 O'Keefe SI, El-Zayadi AR, Carraherte TE, Davis M, Williams R. Malnutrition and immunocompetence in patients with R. Malnutrition and immunocompeter

40 Calvey H, Davis M, Williams R. Controlled trial of nutritiona supplementation with and without branch chain aminoacid enrichment. F Hepatol 1985; 1: 141-51.

41 Bird GLA. Treatment of advanced alcoholic liver disease. Alcohol Alcohol 1990; 25: 197-206.

42 Panos MZ, Polson R, Johnson R, Portmann B, Williams R. Polyunsaturated phosphatidylcholine for acute alcoholic hepatitis. Eur f Gastroenterol Hepatol 1990; 2: 351-5.

43 Bird GLA, O'Grady JG, Harvey FAH, Calne RY, Williams R. Liver transplantation in patients with alcoholic cirrhosis: selection criteria and rates of survival and relapse. $\mathrm{BrMed} \mathcal{F}$ 1990; 301: 15-17.

44 Liver transplantation, Consensus Conference. $\mathcal{F} A M A 1983$; 250: $2961-4$

45 Starzl TE, Van Thiel D, Tzakis AG, et al. Orthotopic liver transplantation for alcoholic cirrhosis. $\mathcal{F} A M A$ 1988; 260; 2542-4.

46 Kumar S, Stauber RE, Gaveler JS, et al. Orthotopic liver transplantation for alcoholic liver disease. Hepatology 1990 11: 159-64. 